\section{Nível de atividade física de professores da cidade de Morro Redondo/RS}

\author{
Physical activity level of teachers from \\ Morro Redondo city
}

Vítor Häfele

Marcelo Cozzensa da Silva ${ }^{1,2,3}$

\section{RESUMO}

O objetivo do estudo foi verificar o nível de atividade física nos diferentes domínios de professores da cidade de Morro Redondo, Rio Grande do Sul. Foi realizado um estudo descritivo do tipo censo em todas as escolas da cidade de Morro Redondo/RS, com exceção da escola de educação infantil. Os dados foram coletados através de um questionário que incluiu questões sobre variáveis econômicas, sociodemográficas, nutricionais, comportamentais e de saúde. O nível de atividade física foi medido através do Questionário Internacional de Atividade Física (IPAQ), versão longa. Foi realizada a análise univariada de todas as informações coletadas, com cálculo das medidas de tendência central e dispersão para as variáveis contínuas e de proporções para as variáveis categóricas. Um total de 73 professores foram entrevistados (98,6\% do total), sendo a média total de AF relatada pelos docentes 1701,7 minutos por semana (DP 1640,0). Quando analisadas as proporções de sujeitos ativos nos domínios da AF de forma dicotômica, verificou-se que 61,6\% apresentaram nível insuficiente de AF no domínio de lazer. $\mathrm{O}$ menor percentual de ativos foi encontrado no domínio do deslocamento (11,0\% dos entrevistados foram considerados suficientemente ativos). Os demais domínios, trabalho e doméstico, apresentaram 19,2\% e 69,9\%, respectivamente, de indivíduos suficientemente ativos. Concluiu-se que professores apresentaram baixos níveis de atividade física nos domínios do lazer, do deslocamento e do trabalho.

\section{PALAVRAS-CHAVE}

Atividade motora; Saúde; Professores; Trabalho.

\begin{abstract}
The aim of the study was to determine the level of physical activity in different domains in teachers from Morro Redondo, Rio Grande do Sul. A census descriptive study was conducted in all schools of the city of Morro Redondo / RS, with the exception of school childhood education. Data were collected through a questionnaire including questions about economic, demographic, nutritional, behavioral and health variables. The physical activity level was analyzed using the International Physical Activity Questionnaire (IPAQ) long version. Univariate analysis of all information collected (of central tendency and dispersion measures for continuous variables and proportions for categorical variables) was performed. A total of 73 teachers were interviewed (98.6\% of the total), and the total PA mean reported was 1701.7 minutes per week (SD 1640.0).Proportion of active subjects in the domains of $P A$ analyzed in two groups found that $61.6 \%$ of the subjects had insufficient levels of $P A$ at leisure time. The lowest percentage of sufficient $P A$ was found in the commuting domain $11.0 \%$ of respondents were considered sufficiently active). Work and domestic domains showed, respectively, $19.2 \%$ and $69.9 \%$ of sufficiently active individuals. It was concluded that teachers had low levels of physical activity in the domains of leisure-time, commuting and work.
\end{abstract}

\section{KEYWORDS}

Motor activity; Health; School teachers; Work.
Rev Bras Ativ Fis Saúde p. 475-483 DOI

http://dx.doi.org/10.12820/rbafs.v.19n4p475

1 Escola Superior de Educação Física, Universidade Federal de Pelotas, Brasil

2 Programa de Pós-Graduação em Educação Física, Universidade Federal de Pelotas, Brasil

3 Grupo de Estudos em Epidemiologia da Atividade Física - Universidade Federal de Pelotas, Brasil 


\section{INTRODUCCÃO}

Ensinar é uma atividade altamente estressante, sendo que seu reflexo na saúde física e mental e no desempenho profissional dos educadores torna-se extremamente relevante ${ }^{1}$. A atividade de ensino envolve, além de ministrar aulas, trabalhos administrativos, planejamento, atualização e investigação constante, orientação de alunos e interação com os pais dos mesmos². Somado a isso, os professores enfrentam algumas dificuldades para a realização de seu trabalho, como estruturas institucionais inadequadas, superlotação nas salas de aula, tempo de intervalo limitado durante o período de trabalho, jornadas de trabalho elevadas e em mais de um turno por dia e baixa remuneração ${ }^{2,3}$. Todos esses fatores tornam-se parte de seu estilo de vida e podem influenciar na qualidade de vida desses profissionais.

O estilo de vida é um dos mais importantes determinantes de saúde dos indivíduos. Dois terços das mortes ocorridas por doenças que podem ser prevenidas e controladas são decorrentes do tabagismo, da alimentação inadequada e da inatividade física ${ }^{4}$. Indivíduos que possuem prática regular de atividades físicas têm benefícios quanto à redução aos riscos de hipertensão arterial, doença coronariana, acidente vascular cerebral, diabetes, câncer de mama e de cólon, depressão e obesidade 5 . A atividade física insuficiente é responsável por cerca de 3,2 milhões de mortes por ano no mundo, constituindo-se assim, como o quarto principal fator de risco para a mortalidade global ${ }^{6}$.

Pesquisa realizada por Florindo et al. ${ }^{7}$ na cidade de São Paulo, mostrou prevalências elevadas de sujeitos insuficientemente ativos em todos os domínios da atividade física ( $91,7 \%$ no deslocamento, $77,5 \%$ no lazer, $68,9 \%$ no trabalho e 56,7\% no domínio doméstico). Quando se tratam de pesquisas sobre o nível de atividade física em professores da Educação Básica, são poucos os estudos encontrados na literatura. Em estudo conduzido com professores escolares da rede pública de uma cidade do sul do Brasil, Santos e Marques ${ }^{8}$ encontraram que $34,8 \%$ dos docentes não atingiram as recomendações mínimas semanais para a obtenção de benefícios à saúde $(150 \mathrm{~min} / \mathrm{sem})$.

Considerando a importância da prática de atividades físicas na prevenção e tratamento de doenças, o escasso número de pesquisas que avaliam esse tema em professores do ensino básico, especialmente, em cidades de pequeno porte e a inexistência de qualquer tipo de investigação científica em professores do município de Morro Redondo, a presente pesquisa teve como objetivo verificar o nível de atividade física nos diferentes domínios de professores da cidade de Morro Redondo, Rio Grande do Sul.

\section{MÉTODOS}

Realizou-se um estudo descritivo do tipo censo, sobre o nível de atividade física nos diferentes domínios de professores da cidade de Morro Redondo/RS.

O município considerado no presente estudo fica localizado na região sul do estado do Rio Grande do Sul e possui, segundo o Instituto Brasileiro de Geografia e Estatística ${ }^{9}$ cerca de seis mil habitantes. A população do estudo foi formada por todos os professores atuantes em sala de aula da cidade de Morro Redondo/RS que lecionavam na Educação Básica, com exceção da creche. A rede de ensino da cidade é constituída por sete escolas públicas (duas 
na zona urbana e cinco na zona rural), sendo uma estadual e seis municipais, com um total de setenta e quatro professores. Todas as escolas da rede e seus professores foram convidados a participar da pesquisa. $\mathrm{O}$ número total de entrevistados foi de setenta e três, visto que houve uma perda devido ao não comparecimento do professor na escola nos dias marcados para entrevista.

Para realizar as entrevistas foi solicitada autorização junto à Secretaria $\mathrm{Mu}$ nicipal de Educação e à $5^{\circ}$ Coordenadoria Regional de Educação. Depois de recebida autorização, fez-se contato com a direção das escolas para a entrega da carta de apresentação do estudo e para agendar as entrevistas com os professores.

A coleta de dados foi realizada no período de outubro a dezembro de 2013, por três entrevistadores treinados pertencentes ao curso de Educação Física da Universidade Federal de Pelotas, os quais passaram por um treinamento de 20 horas. Antes da entrevista o professor assinou o termo de consentimento livre e esclarecido, sendo informado pelo entrevistador sobre os objetivos da pesquisa e também que seria mantido sigilo sobre suas informações.

O questionário utilizado na coleta de dados continha questões medindo características econômicas - renda pessoal (em reais); sociodemográficas sexo (masculino, feminino), idade (anos completos), cor da pele (observada pelo entrevistador), situação conjugal (casado/vive com companheiro, solteiro); comportamentais - tabagismo (fumante, ex-fumante, não fumante), Nível de atividade física (domínios de lazer, deslocamento, doméstico e trabalho); Nutricional -índice de massa corporal (IMC) (medido pelo peso $(\mathrm{kg})$ referi$\mathrm{do}^{10}$, dividido pela altura $(\mathrm{cm})$ referida elevada ao quadrado); De saúde - Auto -percepção de saúde (excelente, muito boa, boa, regular, ruim).

A principal variável de interesse, o nível de $\mathrm{AF}$ em diferentes domínios, foi avaliada através do Questionário Internacional de Atividade Física (IPAQ), versão longa, proposto pela Organização Mundial da Saúde (OMS) e pelo Centro de Controle e Prevenção de Doenças (CDC), e validado em diversos países, incluindo países da América Latina ${ }^{11}$. O referido instrumento é composto por 27 perguntas que medem a prática de atividade física em quatro domínios: trabalho, deslocamento, atividades domésticas e lazer. Foram considerados suficientemente ativos os professores que atingiram, em cada domínio separadamente, 150 minutos ou mais de atividades físicas na última semana ${ }^{12}$.

O banco de dados foi construído no programa Excel e a análise dos dados realizada através do programa STATA 10.0. Foi realizada a análise univariada de todas as informações coletadas, com cálculo das medidas de tendência central (mediana, média e desvio padrão - DP) e dispersão para as variáveis contínuas e de proporções para as variáveis categóricas.

O protocolo do estudo foi submetido e aprovado pelo Comitê de Ética em Pesquisa da Escola Superior de Educação Física da Universidade Federal de Pelotas (protocolo número 607.356 Plataforma Brasil). Os princípios éticos foram assegurados aos professores, de modo que todos consentiram em participar do estudo.

\section{RESULTADOS}

Foram entrevistados 73 professores em sete escolas municipais e estaduais do município de Morro Redondo, havendo somente uma perda. A média de 
idade dos entrevistados foi de 43,3 anos (DP= 10,7 anos); 76,7\% eram do sexo feminino, $58,9 \%$ eram casados/viviam com companheiro e $95,9 \%$ eram de cor da pele branca. A renda média mensal foi de $\mathrm{R} \$ 1828,1$ reais $(\mathrm{DP}=691,5$ reais), sendo que 35,7\% ganhavam até dois salários mínimos. Em relação ao IMC, 59,9\% se encontravam na categoria de sobrepeso/obesidade, sendo o valor médio encontrado de $26,7 \mathrm{~kg} / \mathrm{m}^{2}(\mathrm{DP}=4,8)$. Quando perguntados sobre sua percepção de saúde, $27,4 \%$ disseram percebê-la como excelente/muito boa (Tabela 1).

TABELA 1 - Frequência absoluta (n) e relativa (\%) das variáveis socioeconômicas, demográficas, nutricional e de saúde dos professores da rede pública de ensino da cidade de Morro Redondo/ RS, 2013 ( $N=73)$.

\begin{tabular}{|c|c|c|}
\hline Variáveis & $\mathrm{N}$ & $\%$ \\
\hline \multicolumn{3}{|l|}{ Idade (anos) } \\
\hline $20-29$ & 7 & 9,6 \\
\hline $30-39$ & 23 & 31,5 \\
\hline $40-49$ & 23 & 31,5 \\
\hline 50 ou mais & 20 & 27,4 \\
\hline \multicolumn{3}{|l|}{ Cor da pele } \\
\hline Branca & 70 & 95,9 \\
\hline Não branca & 3 & 4,1 \\
\hline \multicolumn{3}{|l|}{ Sexo } \\
\hline Masculino & 17 & 23,3 \\
\hline Feminino & 56 & 76,7 \\
\hline \multicolumn{3}{|l|}{ Situação conjugal } \\
\hline Solteiro & 30 & 41,1 \\
\hline Casado/vive com companheiro & 43 & 58,9 \\
\hline \multicolumn{3}{|l|}{ Escolaridade } \\
\hline Ensino superior & 30 & 41,1 \\
\hline Especialização & 39 & 53,4 \\
\hline Mestrado/doutorado & 4 & 5,5 \\
\hline \multicolumn{3}{|c|}{ Renda mensal docente (salários mínimos) } \\
\hline Até dois salários & 25 & 35,7 \\
\hline Entre dois e três salários & 18 & 25,7 \\
\hline Mais de 3 salários & 27 & 38,6 \\
\hline \multicolumn{3}{|l|}{ Hábito de fumar } \\
\hline Nunca & 53 & 72,6 \\
\hline Ex-fumante & 17 & 23,3 \\
\hline Fuma & 3 & 4,1 \\
\hline \multicolumn{3}{|l|}{ Percepção de saúde } \\
\hline Excelente & 6 & 8,2 \\
\hline Muito boa & 14 & 19,2 \\
\hline Boa & 43 & 58,9 \\
\hline Regular & 10 & 13,7 \\
\hline \multicolumn{3}{|l|}{ Índice de Massa Corporal (IMC) } \\
\hline Normal & 30 & 41,1 \\
\hline Sobrepeso & 30 & 41,1 \\
\hline Obesidade & 13 & 17,8 \\
\hline
\end{tabular}

Quarenta e seis porcento dos estudados trabalhavam na rede municipal de ensino, sendo que $50,7 \%$ atuavam no ensino fundamental e $64,4 \%$ leciona- 
vam somente em uma escola. O tempo médio de anos trabalhado na escola foi de 16,4 anos (DP 11,6 anos); 86,3\% dos educadores trabalhavam no turno da manhã, 84,9 trabalhavam no turno da tarde e 34,3 no turno da noite $(28,8 \%$ trabalhavam nos três turnos do dia). Mais da metade dos professores $(53,4 \%)$ possuíam curso de especialização e $5,5 \%$ possuíam mestrado/doutorado.

A média de AF relatada pelos trabalhadores durante a jornada de trabalho foi de 169,2 minutos por semana (DP 460,1). A média de tempo despendido em atividades de deslocamento foi de 59,9 minutos por semana (DP 107,0), durante o período de lazer foi de 144,8 minutos por semana (DP 171,1) e em atividades no domínio doméstico foi de 1183,1 minutos por semana (DP 1524,1). O escore médio de AF total relatado pelos participantes foi de 1701,7 minutos por semana (DP 1640,0) (Tabela 2).

TABELA 2 - Nível de AF nos diferentes domínios (medianas, médias e desvios padrão) dos professores de escolas públicas do município de Morro Redondo /RS ( $N=73$ ).

\begin{tabular}{lccc}
\hline DOMíNIO & $\begin{array}{c}\text { MEDIANA } \\
\text { (minutos/semana) }\end{array}$ & $\begin{array}{c}\text { MÉDIA } \\
\text { (minutos/semana) }\end{array}$ & $\begin{array}{c}\text { DESVIO-PADRÃO } \\
\text { (minutos/semana) }\end{array}$ \\
\hline Trabalho & 0 & 169,2 & 460,1 \\
\hline Deslocamento & 20 & 59,9 & 107,0 \\
\hline Lazer & 90 & 144,8 & 171,1 \\
\hline Doméstica & 555 & 1183,1 & 1524,1 \\
\hline TOTAL & 1080,0 & 1701,7 & 1640,0 \\
\hline
\end{tabular}

Quando analisados de forma dicotômica, 61,6\% dos professores foram considerados insuficientemente ativos no lazer (AF $<150 \mathrm{~min} / \mathrm{sem})$. Dentre as atividades praticadas durante o domínio do lazer, 57,5\% dos indivíduos relataram a realização de caminhadas, $31,5 \%$ disseram realizar $\mathrm{AF}$ moderadas e 23,3\% AF vigorosas. As médias de tempo despendidos nas atividades que compõem o cálculo do domínio de lazer estão descritas na Figura 1. Do total de professores, $26,0 \%$ não realizam qualquer tipo de atividade, por no mínimo 10 minutos consecutivos no tempo de lazer (0 minutos/sem).

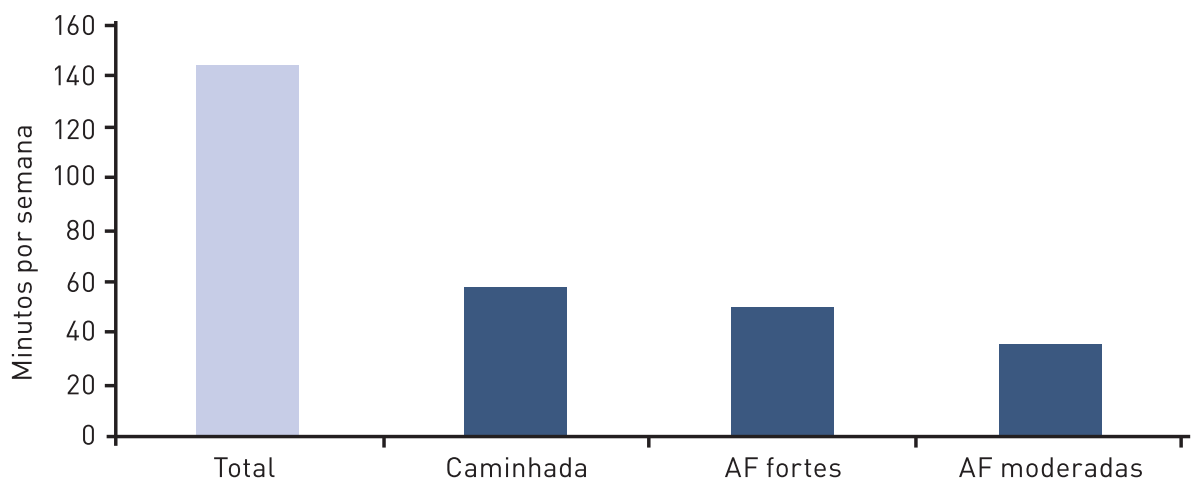

FIGURA 1 - Tipo e tempo médio despendido em atividade física no domínio de lazer em minutos por semana por professores de Morro Redondo / RS ( $N=73$ ).

As menores médias de $\mathrm{AF}$ encontradas entre todos os domínios que compõem o escore geral de AF foram no domínio do deslocamento. Quando analisados, $89,0 \%$ dos professores foram considerados insuficientemente ativos no deslocamento. Importante ressaltar que $42,3 \%$ dos professores relataram não realizar qualquer tipo de atividade nesse domínio por 10 minutos con- 
secutivos. Dos entrevistados que descreveram se deslocar ativamente, 12,8\% relataram se deslocar de bicicleta e $87,2 \%$ através da caminhada.

A maioria dos professores $(69,9 \%)$ atingiu a recomendação mínima de $\mathrm{AF}$ preconizada pela OMS e CDC para obtenção de benefícios à saúde somente no domínio doméstico. As AF moderadas realizadas no pátio ou jardim de suas casas foram as mais relatadas. Já, no domínio do trabalho, somente 19,2\% dos entrevistados conseguiram atingir a recomendação de $150 \mathrm{~min} / \mathrm{sem}$ de AF.

\section{DISCUSSÃO}

A população estudada caracterizou-se como sendo, em sua maioria, do sexo feminino, vivendo em união estável, de cor da pele branca e com média de idade de 43,3 anos. Apesar de serem profissionais do ensino de uma cidade de pequeno porte da região sul do Rio Grande do Sul, suas características não diferem de outros docentes de escolas públicas ${ }^{8,13-15}$ do mesmo e de outros estados do Brasil.

De acordo com a classificação do IMC, mais da metade dos estudados se encontravam na categoria de sobrepeso/obesidade (58,9\%). Este resultado é superior ao encontrado em estudo de Santos e Marques ${ }^{8}$, com professores da educação básica da cidade de Bagé-RS, (46,7\%), de Silva e Silva ${ }^{13}$, com professores pré-escolares de Pelotas-RS $(55,1 \%)$, e de Nascente et al. ${ }^{16}$, com a população de um município de pequeno porte do estado de Goiás (49,7\%). Indivíduos obesos apresentam maior risco de desenvolver hipertensão arterial, resistência à insulina e diabetes mellitus tipo II quando comparados a pessoas com IMC normal ${ }^{17}$.

Em relação ao tabagismo, somente 4,1\% dos docentes entrevistados relataram o hábito de fumo atual. Este resultado é bem inferior ao encontrado em outros estudos com professores escolares ${ }^{8,13,14,18,19}$. De acordo com Barros et al. ${ }^{20}$, profissionais que possuem maior grau de escolaridade, têm praticamente a metade da frequência do consumo de cigarros em relação aos trabalhadores com menor nível educacional.

Os índices sobre a percepção de saúde analisados, assemelham-se com os de outros estudos com professores escolares ${ }^{8,13}$, constituindo-se como um fator positivo, visto que a proporção de sujeitos com percepção de saúde regular foi pequena. Outro fator a ser considerado nesta pesquisa em relação à percepção de saúde, foi o fato dos indivíduos não terem à relatada como ruim.

Não foram encontrados estudos conduzidos no Brasil com professores escolares em relação à verificação de média e mediana de tempo de atividade física total e separada por domínios. Os estudos abordaram apenas o nível suficiente de atividade física de acordo com a recomendação internacional de atividade física, sem levar em conta a média ou mediana de tempo executados ${ }^{8,13,21,22}$.

As atividades físicas realizadas no tempo livre atingem baixas frequências de prática entre a população em geral. $\mathrm{Na}$ mesma direção, o escore de $\mathrm{AF}$ suficiente no lazer encontrado foi baixo, mas superior ao verificado em estudo com professores pré-escolares da cidade de Pelotas ${ }^{13}$ e na população desse município ${ }^{23}$.Já o domínio que apresentou os menores níveis de AF pelos docentes foi o deslocamento. Tal achado se assemelha aos resultados encontrados na população em geral ${ }^{7}$. Uma hipótese para o baixo percentual de ativos nestes dois contextos são os fatores ambientais, visto que a cidade alvo do estudo, não possui pistas exclusivas para ciclistas ou para realização de caminhadas. Aliado 
a isso, a categoria docente possui uma sobrecarga oriunda de uma alta jornada de trabalho em atividades escolares, somadas aos afazeres domésticos ${ }^{24,25}$, fazendo assim, com que esta população tenha pouco tempo para realização de atividades físicas em ambos os domínios.

Outro domínio que apresentou baixas frequências de indivíduos suficientemente ativos foi o ocupacional. Tal resultado segue a tendência encontrada por Silva e Silva ${ }^{13}$, que mesmo utilizando o domínio do trabalho juntamente com o do lazer, encontraram, apenas, pouco mais da metade dos sujeitos ativos. Segundo Silva e Silva ${ }^{13}$, Delcor et al. ${ }^{25}$ e Reis et al. ${ }^{1}$, grande parcela dos professores de escola trabalham constantemente na posição em pé, com pouca movimentação, na maior parte do tempo de aula, o que ajuda a explicar tal resultado.

O domínio doméstico foi o que apresentou maior proporção de sujeitos suficientemente ativos. Este dado se assemelha aos encontrados em outros estudos com diferentes populações, os quais apresentaram elevados percentuais de ativos neste contexto ${ }^{26-28}$. Morro Redondo é um município de pequeno porte (menos de 20 mil habitantes) colonizado por portugueses, alemães e italianos, os quais se caracterizam por sua dedicação ao trabalho, seja ocupacional ou doméstico. Outro fator importe a ser considerado é que quase a totalidade das moradias do município é constituída por domicílios com pátios avantajados, onde há o plantio de frutas e verduras, bem como a criação de animais de pequeno porte. Somado a isso, as tarefas de limpeza da casa, lavagem da roupa e preparo das refeições, entre outras, as quais são predominantemente femininas, ocorrem com frequência diária nesse município. No entanto, pesquisa avaliativa sobre dez anos de utilização do IPAQ em dois países da América latina afirma que o domínio doméstico, é muitas vezes, superestimado pelos entrevistados, devendo seus resultados, portanto, serem interpretados com certa cautela devido à sua imprecisão ${ }^{29}$.

Alguns fatores importantes do presente estudo devem ser destacados. É de conhecimento entre aqueles que pesquisam em atividade física e saúde que as melhores estimativas de atividade física são obtidas através de medidas objetivas. Porém, a aplicação de um questionário validado internacionalmente e aplicado através de entrevistas face a face, ajuda a minimizar erros de informação. Outro ponto a ser salientado é o caráter censitário da pesquisa e o baixo índice de perdas (somente uma).

Considerando os achados do presente estudo, concluiu-se que os professores apresentaram baixos níveis de atividade física nos domínios do lazer, do deslocamento e do trabalho. Entretanto, os mesmos mostraram-se ativos no domínio doméstico, onde a demanda de tempo despendido com cuidados com a casa torna-se importante nesse município. Os resultados obtidos servem de subsídio para o planejamento de estratégias que consigam, de maneira coerente, conduzir os trabalhadores desta classe a níveis suficientes de atividade física para a obtenção de benefícios a saúde.

\section{Contribuição dos autores}

Vítor Häfele coordenou o trabalho de campo, conduziu a revisão de literatura e realizou a escrita do artigo. Marcelo Cozzensa da Silva conduziu a análise de dados, criação de tabelas e figuras e revisão crítica da escrita do artigo. 


\section{Agradecimentos}

Agradecemos à $5^{\circ} \mathrm{CRE}$, a SMEC e as direções das sete escolas de Morro Redondo pela viabilização da pesquisa. A todos os professores que ajudaram na realização deste estudo.

\section{REFERÊNCIAS}

1. Reis EJFB, Araújo TM, Carvalho FM, Barbalho L, Silva MO. Docência e exaustão emocional. Educ Soc. 2006;27:229-53.

2. Mendes MLM. Condições de trabalho e saúde docente. Rio de Janeiro, 2006.

3. Tabeleão VP, Tomasi E, Neves SF. Qualidade de vida e esgotamento profissional entre docentes da rede pública de Ensino Médio e Fundamental no Sul do Brasil. Cad Saúde Pública. 2011;27(12):2401-8.

4. Nahas MV. Atividade física, saúde e qualidade de vida: conceitos e sugestões por um estilo de vida ativo. 4 ed 2006. 284 p.

5. Organizacón Mundial de la Salud. Recomendaciones mundiales sobre actividad física para la salud. 2010.

6. World Health Organization. Global health risks mortality and burden of disease attributable to selected major risks. 2009.

7. Florindo AA, Guimaraes VV, Cesar CL, Barros MB, Alves MC, Goldbaum M. Epidemiology of leisure, transportation, occupational, and household physical activity: prevalence and associated factors. J Phys Act Health. 2009;6(5):625-32.

8. Santos MN, Marques AC. Condições de saúde, estilo de vida e caracteristicas de trabalho de professores de uma cidade do sul do Brasil. Cien Saúde Colet. 2013;18(3):837-46.

9. Instituto Nacional de Geografia e Estatística.IBGE. 2010.

10. World Health Organization. Physical Status:The Use and Interpretation of Anthropometry. 1995.

11. Craig CL, Marshall AL, Sjostrom M, Bauman AE, Booth ML, Ainsworth BE, et al. International physical activity questionnaire: 12-country reliability and validity. Med Sci Sports Exerc. 2003;35(8):1381-95.

12. Haskell WL, Lee IM, Pate RR, Powell KE, Blair SN, Franklin BA, et al. Physical activity and public health: updated recommendation for adults from the American College of Sports Medicine and the American Heart Association. Med Sci Sports Exerc. 2007;39(8):1423-34.

13. Silva LG, Silva MC. Condições de trabalho e saúde de professores pré-escolares da rede pública de ensino de Pelotas, RS, Brasil. Cien Saúde Colet. 2013;18(11):3137-46.

14. Gasparini SM, Barreto SM, Assunção AA. O professor, as condições de trabalho e os efeitos sobre sua saúde. Educ Pesqui. 2005;31(2):189-99.

15. Instituto Nacional de Estudos e Pesquisas Educacionais Anísio Teixeira. INEP. Estudo exploratório sobre o professor brasileiro com base nos resultados do Censo Escolar de 2007. 2009.

16. Nascente FMN, Jardim PCBV, Peixoto MRG, Monego ET, Barroso WKS, Moreira HG, et al. Hipertensão arterial e sua associação com índices antropométricos em adultos de uma cidade de pequeno porte do interior do Brasil. Rev. Assoc. Med. Bras. 2009; 55 (6): 716-722.

17. Organização Mundial da Saúde. Obesidade: previnindo e controlando a epidemia global. São Paulo: Roca; 2004.

18. Jardim R, Barreto SM, Assuncao AA. Condicoes de trabalho, qualidade de vida e disfonia entre docentes. Cad Saúde Pública. 2007;23(10):2439-61.

19. Maciel SSSV, Maciel WV, Silva JM, Sobral LV, Sobral HV, Steinhauser HC, et al. Prevalência do uso de drogas entre professores do ensino médio. Rev Amrigs. 2012;56(4):295-9.

20. Barros AJ, Cascaes AM, Wehrmeister FC, Martinez-Mesa J, Menezes AM. Tabagismo no Brasil: desigualdades regionais e prevalencia segundo caracteristicas ocupacionais. Cien Saúde Colet. 2011;16(9):3707-16. 
21. Brito WF, Santos CL, Marcolongo AA, Campos MD, Bocalini DS, Antonio EL, et al. Physical activity levels in public school teachers. Rev Saúde Pública. 2012;46(1):104-9.

22. Canabarro LK, Neutzling MB, Rombaldi AJ. Nível de atividade física no lazer dos professores de educação física do ensino básico. Rev Bras Ativ Fis Saude. 2011;16(1):11-7.

23. Dumith SC, Domingues MR, Gigante DP. Epidemiologia das atividades físicas praticadas no tempo de lazer por adultos do Sul do Brasil. Rev Bras Epidemiol. 2009;12(4):646-58.

24. Vedovato TG, Monteiro MI. Perfil sociodemografico e condicoes de saude e trabalho dos professores de nove escolas estaduais paulistas. Rev Esc Enferm USP. 2008;42(2):290-7.

25. Delcor NS, Araujo TM, Reis EJ, Porto LA, Carvalho FM, Oliveira e Silva M, et al. Condições de trabalho e saude dos professores da rede particular de ensino de Vitória da Conquista, Bahia, Brasil. Cad Saúde Pública. 2004;20(1):187-96.

26. Bicalho PG, Hallal PC, Gazzinelli A, Knuth AG, Velasquez-Melendez G. Adult physical activity levels and associated factors in rural communities of Minas Gerais State, Brazil. Rev Saúde Pública. 2010;44(5):884-93.

27. Cardoso RK, Rombaldi AJ, Silva MC. Nível de atividade física de coletores de lixo de duas cidades de porte médio do sul do Brasil. Rev Bras Ativ Fis Saude. 2013;18(5):604-613.

28. Hartwig TW, Silva MC, Reichert FF, Rombaldi AJ. Condições de saúde de trabalhadores de academias da cidade de Pelotas-RS: um estudo de base populacional. Rev Bras Ativ Fis Saude. 2012;17(6):500-11.

29. Hallal PC, Gomez LF, Parra DC, Lobelo F, Mosquera J, Florindo AA, et al. Lessons Learned After 10 Years of IPAQ Use in Brazil and Colombia. J Phys Act Health. 2010;7(2):259-64.

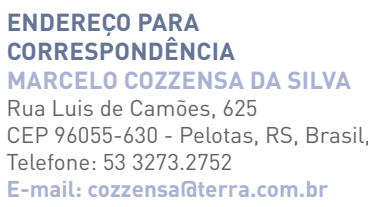

